\title{
Effect of Intractable Epilepsy on the Physiological Cardiac Performance Parameters and the Serum Levels of Cardiac Fatty Acid Binding Protein in Pediatrics
}

\author{
SAAD A. MOHAMED, M.D. ${ }^{\mathbf{1}}$; AHMED ABD EL-TAWAB, M.D. ${ }^{\mathbf{2}}$, MOHAMED MANSOUR, M.D. ${ }^{\mathbf{3}}$; ALAA \\ ABD EL-HAMID, M.D. ${ }^{\mathbf{4}}$; MOHAMMAD SHAFIQ AWAD, M.D. ${ }^{\mathbf{5}}$ and MONA H. TAWFIK, M.D. ${ }^{\mathbf{6}}$ \\ The Department of Pediatrics, Faculty of Medicine, Al-Azhar University, Damietta, Egypt ${ }^{1}$, \\ The Department of Physiology, Faculty of Medicine, Beni-Suef University, Egypt and Jouf University, KSA ${ }^{2}$, \\ The Department of Physiology, Faculty of Medicine, Kasr El-Ainy, Cairo University, Egypt ${ }^{3}$, \\ The Department of Biochemistry, Faculty of Medicine, Beni-Suef University, Egypt and Jouf University, KSA ${ }^{4}$ and \\ The Departments of Cardiology ${ }^{5}$ and Neurology ${ }^{6}$, Faculty of Medicine, Beni-Suef University, Egypt
}

\begin{abstract}
Background: Multiple cardiac changes and various abnormalities have been linked to epileptic fits. The assessment of cardiac changes and serum biomarkers level are commonly accepted methods to evaluate autonomic disturbances during epileptic fits which is very closely associated with some cardiovascular events especially the sudden unexpected cardiac death.
\end{abstract}

Aim of Study: To evaluate ictal and inter-ictal cardiac changes and plasma levels of heart type fatty acid binding protein (H-FABP) in pediatric patients with treatment resistant epilepsy.

Patients and Methods: 32 seizure attacks were recorded in 23 children with intractable seizures. Simultaneous HolterECG and video-EEG recordings were done with evaluation of changes in Heart Rate (HR), parameters of Heart Rate Variability (HRV), as well as serum levels of H-FABP as a marker of myocardial ischemia in the ictal and inter-ictal periods and compared with a control group.

Results: The recorded HR changes included Ictal Tachycardia (IT) (96.87\%), Ictal Bradycardia (IB) (3.13\%), Premature Atrial Contractions (PAC) $(18.75 \%)$ and Premature Ventricular Contractions (PVC) $(28.13 \%)$ in the first postictal hour. Significant HR increase was observed in the preictal hour and ictal values compared to basal values and remained high 2 hours post-ictally. Basal HR and serum levels of the H-FABP protein were significantly higher while time domain HRV parameters were significantly lower in the patients compared to controls.

Conclusion: Significant cardiac changes may occur even before the seizure onset in cases with intractable epilepsy. The increased levels of H-FABP may indicate the presence

Correspondence to: Dr. Mohamed Mansour Khalifa, E-Mail: m mmk2050@yahoo.com of autonomic instability and myocardial ischemia even in asymptomatic patients.

Key Words: Intractable epilepsy - Heart rate - Heart rate variability -EEG - Holter - Heart type fatty acid binding protein.

\section{Introduction}

EPILEPSY is one of the most commonly present types of chronic neurological disease with increased mortality rates among patients with uncontrolled seizures [1]. Changes in the heart rate and rhythm have been proven to be linked to the epileptic attacks, especially Ictal Tachycardia (IT) [2], however, other severe rhythm disorders have been described, including bradycardia, asystole, Bundle Branch Blocks (BBB), ST-segment as well as QTinterval abnormalities [3]. These abnormalities may lead to arrhythmias and/or cardiac ischemia with subsequent sudden cardiac death in epilepsy (SUDEP) that possibly mediated by the autonomic system [4]

Simultaneous ECG-EEG recording during intractable seizures may help to understand ictal semiology, distinguish non-epileptic attacks and disclose SUDEP [5].

When the myocardium is injured, Heart type Fatty Acid Binding Protein (H-FABP) is liberated into the circulation and its serum level is increased, but is usually normalized within 24 hours, that helps to detect recurrent myocardial injury [6]. 
We aimed to evaluate the ictal and inter-ictal cardiac changes in children suffering from intractable epilepsy compared to those findings in healthy controls through simultaneous ECG-EEG recording of seizure episodes and assessment of H-FABP serum levels.

\section{Material and Methods}

Study design: A cross-sectional study was conducted on pediatric cases suffering from intractable epilepsy who were admitted in Pediatric Neurology Department or followed-up at the outpatient clinics at Beni-Suef University Hospital, during the period from August 2015 to February 2017.

Inclusion criteria: Pediatric patients with intractable epilepsy (7) with a history of 2 or more seizure attacks per week, to increase the possibility of recording an ictal attack.

Exclusion criteria: (A) Patients with any evidence of an organic heart diseases or any other diseases that may affect the cardiovascular or autonomic nervous systems, (B) Patients who are currently on any regular medication, apart from the Antiepileptic Drugs (AEDs) and (C) Patients with acute diseases (till cured).

Procedures: Thirty-two seizures episodes were recorded in 23 patients ( 2 attacks were recorded in 9 patients and we omitted attacks that were less than 2 hours apart, to avoid overlapping) and 30 age \& sex-matched healthy controls were included. The patients' plasma levels of H-FABP were withdrawn in 2 samples; the ictal one was within half an hour of the seizure episode while the second (inter-ictal) one was taken when the patient was free of attacks for at least 12-24 hours and compared to the control subjects.

Patients were subjected to a detailed neurological examination and the Chalfont severity scale was used to rate the severity of the seizure [8]

Recording was synchronized at the onset including video-recorded EEG (Xltek, UltraPro S 100, UK) (to document an ictal event) simultaneously with Holter-ECG (DR200/HE, Tel-a-heartTM, USA) for a total period of 6 hours. Clinical observation and the video images help to identify seizures' semiology, the onset and the termination of the attacks. In one seizure episode, the mother described abnormal jerky movements that were not epileptic by the EEG recording. The parents were then asked to avoid any condition that might affect the mood of the patient or induce stress.

Retrospective analysis of the Holter-ECG recordings was done in the basal recording, pre-ictal hour, ictal period and in the first and the second post-ictal hours. Basal values were evaluated after elimination of the pre-ictal hour, ictal period and the 2 post-ictal hours. It included analysis of:

- Mean values of HR where IT and IB were considered when there was at least a $10 \%$ change (increase or decrease) than the basal HR [9] .

- Time domain measurements of HRV [10] including: SDNN (ms): Standard deviation of all the normal RR intervals in the whole 24-h ECG recording period; SDANN (ms): Standard deviation of the averaged sinus RR intervals for all 5minutes segments of the whole recording; SDNNI (ms): Mean values of the standard deviations of all normal RR intervals for all the interval of 5 minutes segments of the whole recording; rMSSD (ms): Root mean squares of the successive RR intervals difference (the square root of the mean of the sum of the squares of the differences between the adjacent RR intervals over the whole recording); and pNN50 (\%): The percentage of difference between the adjacent RR intervals which are greater than $50 \mathrm{~ms}$ duration computed over the 24-h ECG recording.

- Corrected QT (QTc) according to the Bazett's formula; $[\mathrm{QTc}=\mathrm{QT}$ measured / squared root RR interval].

- Other recorded peri-ictal arrhythmias as: PAC, PVC and ST segment changes.

Frequency domain measurements of the HRV were not supported by the holter set. The measurement of the control group included the average HR, QTc intervals and time domains of the HRV over a period of six hours.

The serum H-FABP levels were measured using 2 venous blood samples, each $3 \mathrm{~mL}$, and the serum was centrifuged at $1000 \mathrm{xg}$ for 10 minutes and was then stored at $-20^{\circ} \mathrm{C}$ till the assay of H-FABP by ELISA technique.

Health education for both the parents and the cooperative patients was conducted separately including emotional support and emphasis on the importance of regular medication, follow-up at outpatient clinics including cardiology clinic, notification of any behaviors changes, cooperation with school teachers for monitoring of academic performance and rehabilitation. 


\section{Ethical consideration:}

The study was approved by the medical local research ethical committee and a written informed consent was taken from the parents with ensuring both the confidentiality and privacy throughout the study process.

\section{Statistical analysis:}

Statistical analysis was performed using the SPSS computer software program package Version 21.0 (SPSS Inc., Chicago, IL, USA). The mean \pm $\mathrm{SD}$ was used for the quantitative variables and the number $(\%)$ for the qualitative variables. Independent samples $t$-test was applied to test for the differences between the means of the quantitative variables between patients and controls. The relation between the quantitative variables within the patient group was analyzed using the paired samples $t$ test while Mann-Whitney $U$-test \& the Wilcoxon Signed test were used for non-parametric statistics. Pearson's correlation coefficient was used to perform the correlation analysis among the various quantitative variables within the case group. The statistical methods were verified by assuming the significance level of $p<0.05$.

\section{Results}

The study included 23 pediatric patients with intractable epilepsy; 14 males and 9 females with a mean age $7.4 \pm 4.6$ (range $2.5-14$ ) years. The underlying etiology included hypoxic ischemic encephalopathy (in 14 patients), cerebro-vascular accident (in 6 patients) and post meningoencephalitis sequelae (in 3 patients). With a mean duration of illness was 5.4 \pm 3.2 (range 2-11.5) years, and the mean duration of recorded seizures was $44.6 \pm 41.9$ (range 6-140) seconds and the average Chalfont severity score was $31.0 \pm 9.5$ (range 9-48).

Thirty two seizure episodes (19 focal and 13 generalized) were recorded during the study period. In focal seizures, $13(68.4 \%)$ showed secondary generalization, $6(31.6 \%)$ showed a temporal origin in EEG recording and 13 had extra-temporal ones.

Different regimens of AEDs were used and changed by the patients according to each case. At the recording time: 11 patients were on two AEDs, 7 on three AEDs, and 5 on four AEDs. The drugs included valproic acid, phenoparbital, clonazepam, lamotrigine, carbamazepine, levetiracetam, topiramate, phenytoin and oxcarbazepine (Table 1).
Table (1): Description of seizures.

\begin{tabular}{|c|c|c|}
\hline Variable & No. & $\%$ \\
\hline \multicolumn{3}{|l|}{ Type of seizures: } \\
\hline Focal & $19 / 32$ & 59.4 \\
\hline Generalized & $13 / 32$ & 40.6 \\
\hline \multicolumn{3}{|l|}{ Localization of focal seizures: } \\
\hline Temporal & $6 / 19$ & 31.6 \\
\hline Extra-temporal & $13 / 19$ & 68.4 \\
\hline \multicolumn{3}{|l|}{ Etiology of seizures: } \\
\hline Hypoxic ischemic encephalopathy & $14 / 23$ & 60.9 \\
\hline Cerebro-vascular accident & $6 / 23$ & 26.1 \\
\hline Post meningo-encephalitis & $3 / 23$ & 13.0 \\
\hline \multicolumn{3}{|l|}{ AEDs regimen: } \\
\hline 2 AEDs & $11 / 23$ & 47.8 \\
\hline 3 AEDs & $7 / 23$ & 30.5 \\
\hline 4 AEDs & $5 / 23$ & 21.7 \\
\hline \multicolumn{3}{|l|}{ Duration of illness (years): } \\
\hline Mean \pm SD & \multicolumn{2}{|c|}{$5.4 \pm 3.2$} \\
\hline Range & \multicolumn{2}{|c|}{$2-11.5$} \\
\hline \multicolumn{3}{|l|}{ Duration of seizure attacks (sec)*: } \\
\hline Mean \pm SD & \multicolumn{2}{|c|}{$44.6 \pm 41.9$} \\
\hline Range & \multicolumn{2}{|c|}{$6-140$} \\
\hline \multicolumn{3}{|l|}{ Chalfont severity score: } \\
\hline Mean \pm SD & \multicolumn{2}{|c|}{$31.0 \pm 9.5$} \\
\hline Range & \multicolumn{2}{|c|}{$9-48$} \\
\hline
\end{tabular}

\section{Regarding HR and rhythm changes:}

- IT was detected in $31 / 32$ of the recorded seizures $(96.87 \%)$, IB in $1 / 32(3.13 \%)$, PACs in $6 / 32$ $(18.75 \%)$ and PVCs in $9 / 32(28.13 \%)$ of seizure episodes. Both PACs and PVCs occurred in the first post-ictal hour. Fig. (1).

- The mean values of basal HR in the patients' group (81.2 $\pm 10.3 \mathrm{bpm})$ were significantly lower than their values in the control group $(95.8 \pm 8.2$ bpm) $(t=5.7, p<0.001)$.

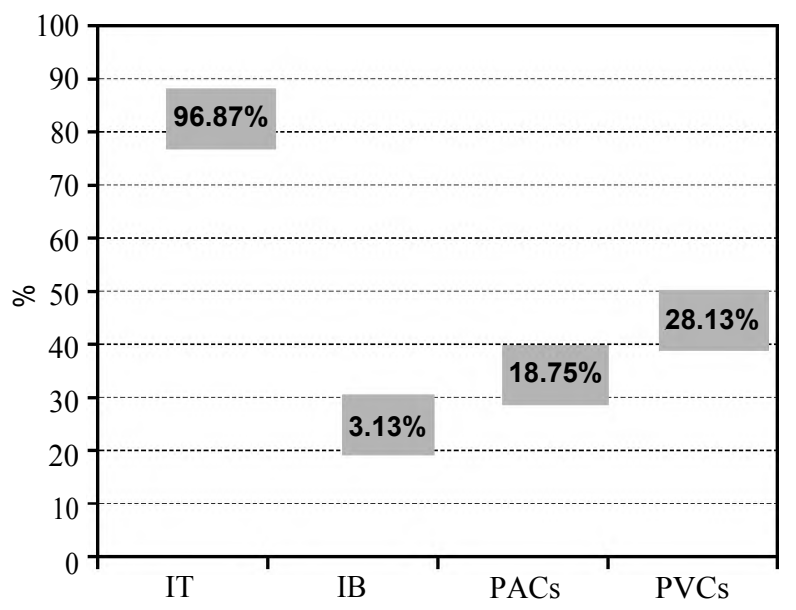

Fig. (1): Percent distribution of patients according to rhythm abnormalities during the recorded seizures. 
- Within the patients' group, the mean ictal HR was significantly higher than the basal values and the first pre-ictal hour $(\mathrm{z}=4.2, p<0.001 \& \mathrm{z}=$ $3.7, p<0.001$, respectively).

- In the first and second post-ictal hours, the mean HR was significantly lower than the ictal value ( $\mathrm{z}=3.5$ and $p<0.001$ and $\mathrm{z}=4.0$ and $p<0.001$ respectively) but still significantly higher than the basal values. (Table 2 ).

- The mean basal values of QTc durations were not significantly different in the patients and control groups $(t=1.69, p=0.097)$. Prolonged QTc values $(>440 \mathrm{~ms}$ ) were recorded in $5 / 23$ patients (21.7\%). (Table 2).

- The mean ictal HR showed no significant correlations with either the duration of illness $(r=0.27$,
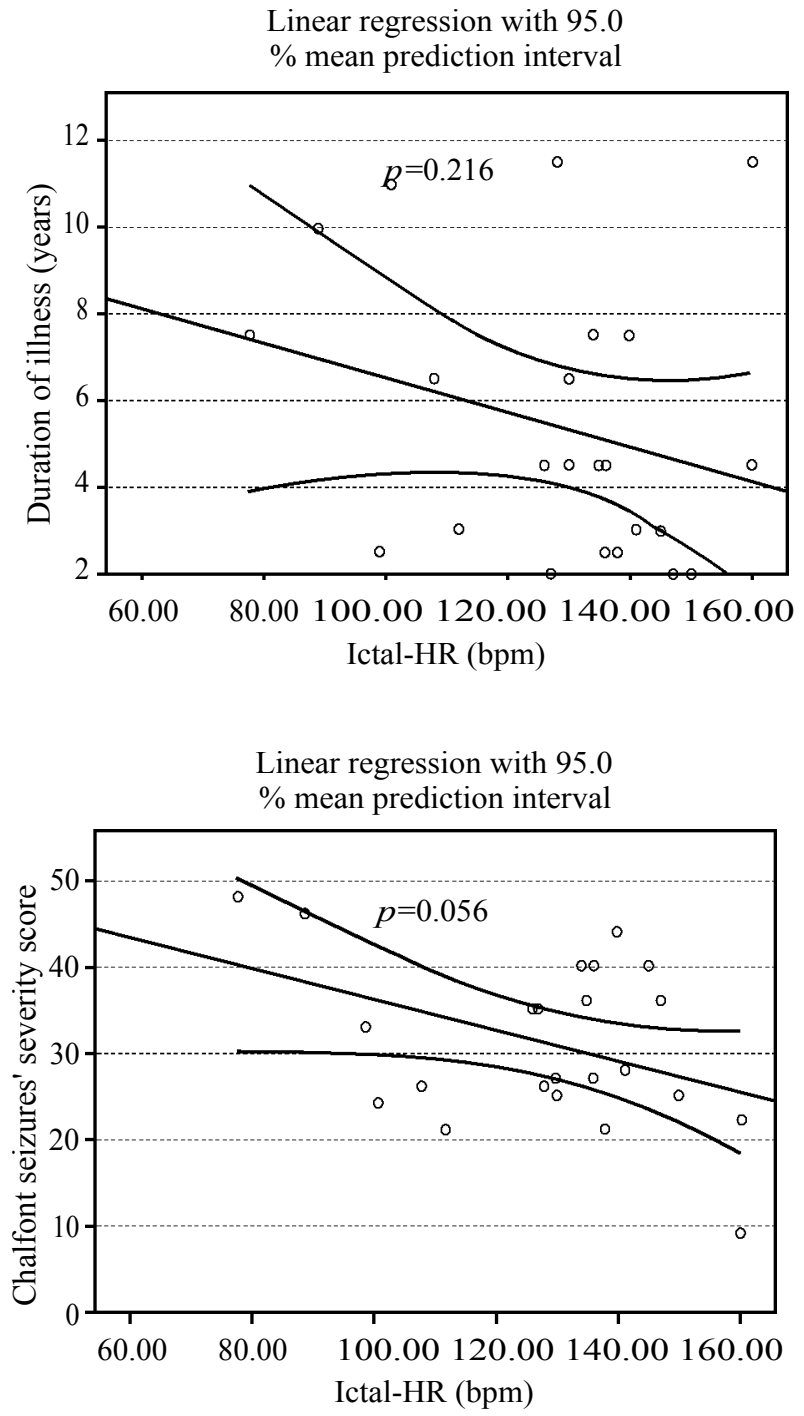

$p=0.216)$, duration of recorded seizure attacks $(r=-0.25, p=0.247)$ or Chalfont seizures' severity score $(r=-0.41, p=0.056)$. Fig. (2).

Table (2): Relations of the heart rate and the corrected QT interval in patients and controls.

\begin{tabular}{|c|c|c|}
\hline Variable & $\begin{array}{l}\text { Patients } \\
(\mathrm{M} \pm \mathrm{SD})\end{array}$ & $\begin{array}{c}\text { Controls } \\
(\mathrm{M} \pm \mathrm{SD})\end{array}$ \\
\hline $\begin{array}{l}\text { Heart rate }(\mathrm{bpm}) \text { : } \\
\text { Basal } \\
\text { First pre-ictal hour } \\
\text { Ictal } \\
\text { First post-ictal hour } \\
\text { Second post-ictal hour }\end{array}$ & $\begin{array}{l}81.2 \pm 10.3 \\
108.6 \pm 17.7 \\
128.2 \pm 21.4 \\
110.9 \pm 10.8 \\
97.9 \pm 8.6\end{array}$ & $95.8 \pm 8.2$ \\
\hline Basal QTc (ms) & $438.6 \pm 4.4$ & $436.5 \pm 4.1$ \\
\hline
\end{tabular}

- Significant difference compared to:

${ }^{1}$ The controls value, ${ }^{2}$ The basal value.

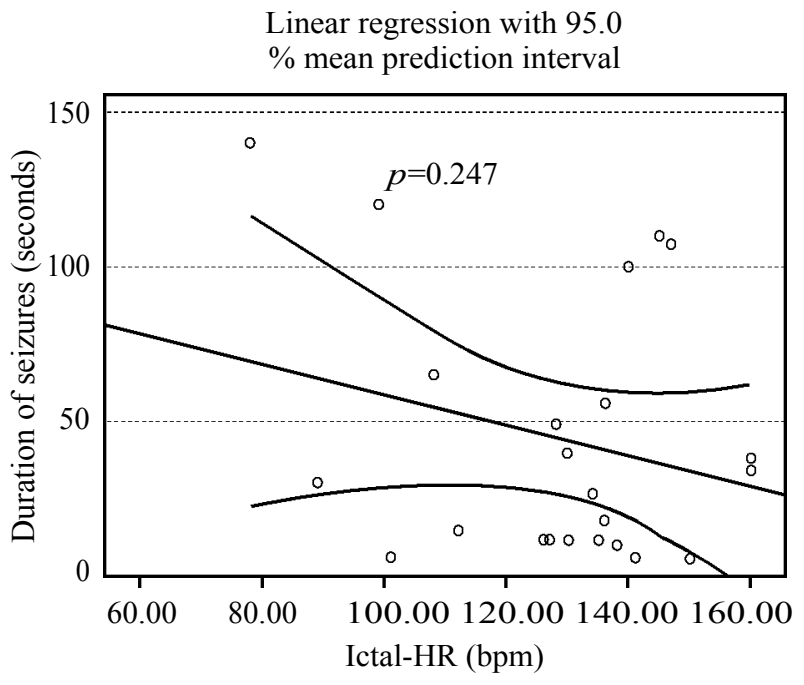

Fig. (2): Correlations between the mean ictal HR and duration of illness, duration of recorded seizures and Chalfont seizures' severity score. 
Regarding time domain measures of HRV (SDNN, SDANN, SDNNI, pNN50 and rMSSD):

- All basal values in the patients' group were statistically significantly lower than that values in the control group $(t=5.4,5.1,4.3,7.7$, and 5.8 respectively and $p<0.001$ in all).

- In the pre-ictal hour, the mean values of SDNN, SDANN, $p$ NN50 and rMSSD of the patients were significantly lower than their basal values $(t=4.3$, $p=0.001 ; t=2.5, p=0.031 ; t=9.0, p<0.001 ; t=5.1$, $p<0.001$ respectively).

- In the first post-ictal hour, the mean values of SDNN, SDANN, SDNNI, and pNN50 of the patients were significantly lower than their basal values ( $t=6.3,6.4,4.8$ and 15.8 respectively and $p<0.001$ in all) and as well than the pre-ictal values $(t=5.5,9.0,5.1$ and 11.0 respectively and $p<0.001$ in all).

- In the 2 nd post-ictal hour, the mean values of SDNN, SDANN, SDNNI, pNN50 and rMSSD of the patients were significantly lower than their basal values $(t=3.5, p=0.005 ; t=5.6, p<0.001$; $t=3.7, p=0.004 ; t=6.8, p<0.001$ and $t=6.2, p<0.001$ respectively) and as well than the pre-ictal values $(t=2.6, p=0.027 ; t=4.6, p=0.001 ; t=2.8, p=0.018$; $t=3.1, p=0.010$ and $t=3.8, p=0.003$ respectively).

- There was no statistically significant difference between the mean values of HRV in the 1 st and the 2 nd post-ictal hours. (Table 3 ).

Table (3): Relations of the heart rate variability in patients and controls.

\begin{tabular}{lccccc}
\hline & \multicolumn{4}{c}{ Patients } & \\
\cline { 2 - 5 } HRV & Basal & $\begin{array}{c}\text { Pre-ictal 1 } \\
\text { hour }\end{array}$ & $\begin{array}{c}\text { 1st post-ictal 1 } \\
\text { hour }\end{array}$ & $\begin{array}{c}\text { 2nd post-ictal 1 } \\
\text { hour }\end{array}$ & Controls \\
\hline SDNN (ms) & $91.8 \pm 17.0^{1}$ & $84.8 \pm 15.8^{\mathbf{2}}$ & $77.2 \pm 14.9^{\mathbf{2 , 3}}$ & $78.1 \pm 15.5^{\mathbf{2 , 3}}$ & $118.5 \pm 18.0$ \\
SDANN (ms) & $72.0 \pm 5.8^{1}$ & $68.4 \pm 5.9^{\mathbf{2}}$ & $60.5 \pm 7.0^{\mathbf{2 , 3}}$ & $62.8 \pm 6.3^{\mathbf{2 , 3}}$ & $95.7 \pm 15.8$ \\
SDNNI (ms) & $43.9 \pm 9.5^{1}$ & $41.6 \pm 8.2$ & $34.8 \pm 10.6^{\mathbf{2 , 3}}$ & $34.6 \pm 9.4^{\mathbf{2 , 3}}$ & $70.6 \pm 18.2$ \\
pNN50 (\%) & $11.5 \pm 4.3^{1}$ & $8.8 \pm 4.2^{\mathbf{2}}$ & $5.3 \pm 3.8^{\mathbf{2 , 3}}$ & $5.8 \pm 4.0^{\mathbf{2 , 3}}$ & $28.6 \pm 8.1$ \\
rMSSD (ms) & $35.4 \pm 7.1^{1}$ & $30.8 \pm 7.2^{\mathbf{2}}$ & $28.7 \pm 10.3$ & $26.4 \pm 5.9^{\mathbf{2 , 3}}$ & $60.3 \pm 15.4$ \\
\hline Significant difference compared to: & ${ }^{\mathbf{1}}$ The controls value, ${ }^{\mathbf{2}}$ The basal value, ${ }^{\mathbf{3}}$ The pre-ictal one hour value.
\end{tabular}

\section{Regarding H-FABP serum levels:}

- The mean serum level of H-FABP (pg/ml) were significantly higher in the patients' group in both ictal $(1374.7 \pm 655.0)$ and inter-ictal phases (1059.1 $\pm 455.7)$ than in the control group $(530.6 \pm 103.0)$ $(t=6.9$ and 6.1 respectively and $p<0.001$ in both).

- The mean serum levels of H-FABP in the patients' group were not significantly different in the ictal and inter-ictal phases $(\mathrm{z}=1.7, p=0.088)$ Fig. (3).

- There was no statistically significant correlation between H-FABP serum levels in the ictal/interictal phases with either the duration of illness, the duration of the recorded seizures, or the Chalfont severity score of the patients $(p>0.05$ in all).
Ictal X controls, $p<0.001$ Interictal X controls, $p<0.001$ Ictal $\mathrm{X}$ interictal, $p=0.088$

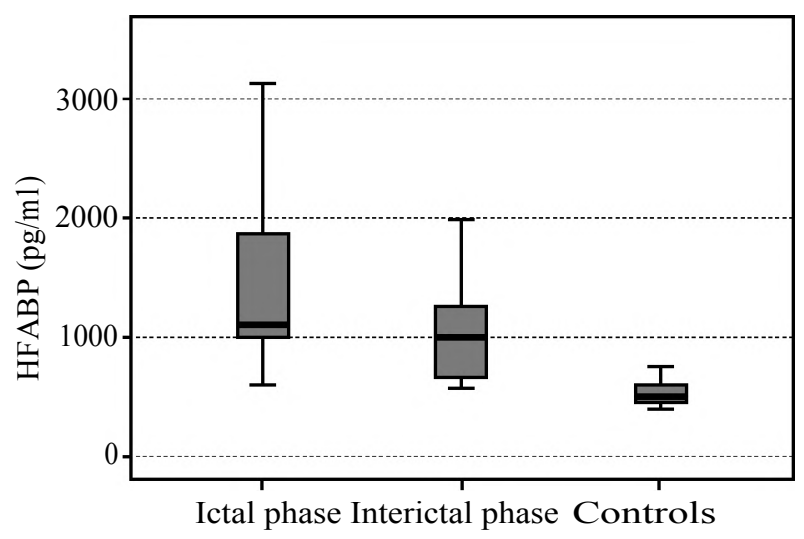

Fig. (3): Ictal and inter-ictal levels of H-FABP of patients. 


\section{Discussion}

Epileptic patients are at a higher risk of cardiac arrhythmias as there is a relationship between the ventricular recovery and the vulnerability of arrhythmias. Early recognition of cardiac changes would help in predicting seizures and better individualized management protocols [11].

No clear parameters can predict the patients who are at an increased risk for SUDEP. So no clear clinical recommendations for the prophylactic therapy are currently present, however recent studies correlate episodes of autonomic instability as well as sudden attacks of seizures with SUDEP [44].

\section{Heart rate changes:}

Heart rate changes occur in most seizure attacks, thought they were most commonly transient and have no fatal effects, however, recent reports of sudden death during or shortly following epileptic fits raised the concerns and started to direct the research interests toward studying the changes in heart rate related to the fits [45]

Several recent researchers examined the heart rate changes related to epilepsy in adult, however few studies examined such relationship in children. As the Nova scotia study. Which found a higher incidence of death in children suffering from epilepsy compered to age matched normal children [46].

The mean values of basal HR in our results showed a statistically significant decrease in the patients in comparison to the control group. This may suggest a sustained dysfunction of autonomic control of HR [5]

Tachycardia can either precede, coincide or follow ictal discharges [12]. Ictal tachycardia was the most prevalent cardiac change noticed in our study during epileptic fits $(96.87 \%$ of recorded seizures) and that was similar to that of previous studies $[\mathbf{5 , 1 3}]$.

Many SUDEP victims are found in bed at the time of death, denoting that some of the causative mechanisms including autonomic instability may occur during sleep. However, studies suggest that a seizure attack maybe also responsible for such death [43]

Changes in HR, as evidenced by simultaneous ECG and EEG recording in our study, precede both clinical seizures and electrical discharge. Similar results were reported by Sevcencu and Struijk [14]
The value of this finding is related to seizure detection even before its clinical onset. On the other hand, the HR showed significant elevation above the basal values that continued for up to 2 hours post-ictally. This was in accordance with other studies that assessed peri-ictal HR at variable durations from the ictal event and IT was reported to be continued even, up to 3 hours post-ictally that might predict fatal arrhythmias $[15,16]$.

Recent researches suggests that simultaneous EEG and ECG recordings revealed that seizure related cardiac rate and rhythm changes frequently outlasts the abnormal brain electrical activity, and may even preceeds the ictal pattern obtained by a scalp EEG recording, and may persist for several minutes to hours after the seizure, suggesting a role for such autonomic changes as a predisposing factor for fatal arrhythmias [47].

Ictal bradycardia was recorded in only one seizure episode (3.13\% of seizures) and this was similar to findings reported by other authors $[5,13]$ Bradyarrhythmia are less frequently found in seizures of various origins and could be a contributing factor in SUDEP [17]. Schuele et al., suggested that asystole or short duration bradycardia might be a benign condition [18], however, Surges et al., raised attention that longer episodes (e.g., tens of seconds to minutes) could lead to a fatal cerebral hypoxemia or a sudden cardiac death [19]

Also if additional seizures occur following a previous one, but before the heart rate returns to its baseline value, there can be incremental heart rate increases associated with an increased variability pattern as well as more frequent abnormal electrical activity patterns associated with each subsequent seizure occurring within this period. Which suggest that significant arrhythmias might occur late post seizure and may have fatal clinical consequences [48].

Postmortem examinations of cardiac tissue in individuals who died from SUDEP have revealed evidence of irreversible pathologic changes, including interstitial fibrosis, myocyte vacuolization, endothelial dysfunction and perivascular infiltration which might be a result of an increased adrenergic output associated with fits. It is postulated that recurrent seizure related injury may cause cardiac fibrosis over time. Which may serve as a focus increasing the risk of cardiac arrhythmias due to increased sympathetic stimulation during subsequent seizures [51] 


\section{Heart rate variability $(H R V)$ changes:}

Our results showed a statistically significant reduction in the basal values of HRV in the patients group compared to the control group. These results were supported by the findings reported in some previous studies in both children and adults $[17,20,21]$. HRV reflects the beat to beat changes in the HR and is mainly modified by the autonomic activity. It may be associated with susceptibility to cardiac arrhythmias and even to be a predictor of mortality after acute myocardial infarction [22]

Our results showed a progressive reduction in time domain measurements of HRV in the periictal periods, possibly resulting from parasympathetic or vagal tone reduction. This reduction might contribute to an increase in the excitability of ventricular conduction system making patients more liable to arrhythmias and possibly susceptible to sudden unexpected death in epilepsy [23]. Hirsch et al., suggested that prolonged/repeated ictal activity might contribute to malignant dysrhythmias [24].

\section{QTc and cardiac arrhythmic changes:}

Another risk factor that might contribute to life-threatening ventricular cardiac arrhythmia and sudden unexpected cardiac death is QTc abnormalities in epileptic patients [25]. Prolonged/shortening QT interval might affect cardiac polarization and accordingly predispose to arrhythmia and sudden death [26,27]. About one fifth of our patients showed prolonged QTc values (>440ms). On the contrary, there was no statistically significant difference between the patient and control groups regarding the mean basal QTc values. Similar findings were reported by other authors $[\mathbf{5 , 1 3}]$ while other authors reported similar QTc interval durations in both groups [28,29]. However, a significant increase in QTc values were reported in other studies among epileptic patients compared to the control group [30,31]

Prolongation of QTc in epileptic patients might be related to the site of ictal activity in the brain [32], genetic mutations of some genes encoding for ion transport channels which are responsible for controlling the ventricular repolarization process (cardiac channelopathies) [33,34], use of QT interval prolonging or depolarization blocking drugs [31], release of catecholamines [35] and ictal hypoxemia and hypercapnia $[\mathbf{4 , 3 6}]$.

Six of our patients had PACs and 7 of them showed nine PVCs that were recorded in the 1 st post-ictal hour. No other ECG changes were recorded. However, cardiac rhythm and ECG abnor- malities in the cases of intractable epilepsy are well-known phenomena including; ST segment abnormalities, asystole, atrial fibrillation, sinus arrhythmia, supraventricular tachycardia, abnormal QRS intervals, junctional escape and bundle-branch block [37-41]

Blumhardt et al., reported that $92 \%$ of patients with seizures recorded by ambulatory EEG-ECG monitoring were associated with a marked increase in heart rate [49]. Subsequently, Smith et al., found that the most common pattern of heart rate change associated with complex partial seizures is that of an initial steep acceleration at the onset of the seizure, followed by marked variatiability pattern during the seizure and postictally [50]

\section{Serum H-FABP levels changes:}

Ischemic injury may occur in treatment resistant epilepsy. The mechanism may be related to excessive autonomic stimulation, which if prolonged, may result in the development of cardiac arrhythmia as well as structural damage to the heart, increasing the susceptibility to cardiac ischemia. Myocardial fibrosis has been reported in cases of treatment resistant epilepsy [52]

Though most patients with uncomplicated epileptic seizures do not seem to have elevated levels of cardiac Troponin postictally, however, signs of ischemia on ECG and elevated other cardiac enzymes in these patients suggest secondary cardiac damage related to epilepsy $[\mathbf{5 3 , 5 4}]$.

Alehan et al., could show the presence of elevated BNP and CK-MB in patients with seizures, the first evidence of subtle cardiac dysfunction in epilepsy patients [55]

As a newly discovered marker of myocardial ischemia, H-FABP has showed more sensitivity and specificity than older markers as troponin I, Brain-type natriuretic peptide and CK-MB even in the absence of frank necrosis with recent evaluation in patients with intractable epilepsy $[\mathbf{5 , 1 3 , 4 2}]$ The values of H-FABP in our patients showed a significant increase in both the ictal and the interictal phases compared to the control group. Ictal serum levels of H-FABP were increased compared to their inter-ictal levels, but this difference didn't reach the statistically significant level. This might be explained by the chronic state of myocardial ischemia in our patients, the narrow intervals between ictal and inter-ictal periods in addition to the small number of our patients with large variability of data [5] 
No significant correlation was found between either duration of illness, duration of recorded seizures or Chalfont severity score of the patients with H-FABP levels in both inter-ictal and ictal phases. The small and heterogeneous group of patients with multiple seizures, the chronic nature of epilepsy disorder and variation in age, origin, duration and time of seizures may have biased our data and might explain the lack of possible effects of those parameters. Similar results were reported by other authors $[5,13]$.

In conclusion, significant cardiac and serum $\mathrm{H}-\mathrm{FABP}$ changes associated with ictal and interictal events provide a promising as well as a noninvasive biomarker for the detection of autonomic instability during seizures and possible prediction of cardiac complications. These abnormalities are expected more frequently in patients with intractable epilepsy and their recording and assessment could help the precision of an individual SUDEP risk assessment and theoretically improve the quality of life and treatment efficiency.

The limitations of our study included: The relatively small number of patients and recorded seizures which might explain the lack of significant effect of the studied parameters, lack of detailed previous information regarding some possible risk factors for SUDEP such as ECG abnormalities, cause of seizures, the doses of the used antiepileptic drugs and its serum levels, and finally lack of more specific time frames evaluation of different parameters in the few seconds and minutes before and after ictal seizures.

Acknowledgement: The authors would like to thank all participating technicians, mothers and children for their help and cooperation.

Conflict of interest: The authors declare that they have no conflict of interest.

\section{References}

1- SILLANPÄÄ M. and SHINNAR S.: Long-term mortality in childhood-onset epilepsy. N. Engl. J. Med., 363 (26): 2522-9, 2010

2- NILSEN K., HARAM M., TANGEDAL S., SAND T. and BRODTKORB E.: Is elevated pre-ictal heart rate associated with secondary generalization in partial epilepsy? Seizure, 19 (5): 291-5, 2010.

3- STANDRIDGE S.M., HOLLAND K.D. and HORN P.S.: Cardiac Arrhythmias and Ictal Events Within an Epilepsy Monitoring Unit. Pediatr. Neurol., 42 (3): 201-5, 2010.

4- GOLDMAN A.M.: Mechanisms of sudden unexplained death in epilepsy. Curr. Opin. Neurol., 28 (2): 166-74, 2015.
5- TOMOUM H.Y., ALY R.H., YOUSSEF O.I., ABDELAL H., NOUR M.U. and EL-SHARKASY A.H.: Heart type fatty acid binding protein, a marker of myocardial ischemia in children with epilepsy. J. Pediat. Neurol., 11 (3): 14957, 2013.

6- AKBAL E., ÖZBEK M., GÜNEŞ F., AKYÜREK Ö., ÜRETEN K. and DELIBAŞ1 T.: Serum heart type fatty acid binding protein levels in metabolic syndrome. Endocrine, 36 (3): 433-7, 2009.

7- ANEJA S. and JAIN P.: Refractory Epilepsy in Children. Indian J. Pediatr., 81 (10): 1063-72, 2014.

8- DUCAN J.S. and SANDER J.W.: The Chalfont seizures severity score: J. Neutrol. Neurosurg. Psychiatry, 54 (10): 873-6, 1991.

9- ISIK U., AYABAKAN C., TOKEL K. and ÖZEK M.: Ictal electrocardiographic changes in children presenting with seizures. Pediatrics International, 54 (1): 27-31, 2012.

10- CYGANKIEWICZ I. and ZAREBA W.: Heart Rate Variability. In: Handbook of Clinical Neurology. Buijs RM, Swaab DF (Eds.), 3 rd edition, Elsevier B.V., 117 (31): 379-94, 2013.

11- YAMASHITA S., YOSHIDA A., FUKUZAWA K., NAKANISHI T., MATSUMOTO A., KONISHI H., et al.: The relationship between cardiac vulnerability and restitution properties of the ventricular activation recovery interval. J. Cardiovasc. Electrophysiol., 26 (7): 768-73, 2015.

12- EGGLESTON K.S., OLIN B.D. and FISHER R.S.: Ictal tachycardia: The head-heart connection. Seizure, 23 (1): 496-505, 2014.

13- EL-SHORBAGY H.H., ELSAYED M.A., KAMAL N.M., AZAB A.A., BASSIOUNY M.M. and GHONEIM I.A.: Heart-type fatty acid-binding protein as a predictor of cardiac ischemia in intractable seizures in children. $\mathbf{J}$. Pediatr. Neurosci., 11 (3): 175-81, 2016.

14- SEVCENCU C. and STRUIJK J.: Autonomic alterations and cardiac changes in epilepsy. Epilepsia, 51 (5): 725 37, 2010.

15- OLIVEIRA G., GONDIM F., HOGAN E. and ROLA F.: Movement-induced heart rate changes in epileptic and non-epileptic seizures. Arq. Neuro-Psiquiatr., 67 (3): 789 91, 2009.

16- TOTH V., HEJJEL L., KALMAR Z., FOGARASI A., AUER T., GYIMESI C., et al.: Effect of epileptic seizures on the heart rate. Ideggyogy Sz., 61 (5-6): 155-61, 2008.

17- CHRONI E., SIRROU V., TRACHANI E., SAKELLAROPOULOS G.C. and POLYCHRONOPOULOS P.: Interictal alterations of cardiovagal function in chronic epilepsy. Epilepsy Res., 83 (2-3): 117-23, 2009.

18- SCHUELE S., BERMEO A., LOCATELLI J., BURGESS R. and LUDERS H.: Ictal asystole: A benign condition? Epilepsia, 49 (1): 168-71, 2008.

19- SURGES R., THIJS R., TAN H. and SANDER J.: Sudden unexpected death in epilepsy: Risk factors and potential pathomechanisms. Nat. Rev. Neurol., 5 (9): 492-504, 2009.

20- EL-SAYED H., KOTBY A., TOMOUM H., EL-HADIDI E., EL-BEHERY S. and EL-GANZORY A.: Non-invasive 
assessment of cardioregulatory autonomic functions in children with epilepsy. Acta. Neurol. Scand., 115 (6): 377-84, 2007.

21- CHEN W., GUO C., ZHANG P., LIU C., QIAO H., ZHANG J., et al.: Heart rate changes in partial seizures: Analysis of influencing factors among refractory patients. B.M.C. Neurol., 14: 135-45, 2014.

22- JANSEN K. and LAGAE L.: Cardiac changes in epilepsy. Seizure, 19 (8): 455-60, 2010.

23- HARNOD T., YANG C.C., HSIN Y.L., SHIEH K.R., WANG P.J. and KUO T.B.: Heart rate variability in children with refractory generalized epilepsy. Seizure, 17 (4): 297-301, 2008.

24- HIRSCH L.J., DONNER E.J., SO E.L., JACOBS M., NASHEF L., NOEBELS J.L., et al.: Abbreviated report of the NIH/NINDS workshop on sudden unexpected death in epilepsy. Neurology, 76 (22): 1932-8, 2011.

25- TEH H., LOO C., TAN H. and RAYMOND A.: The QT interval in epilepsy patients compared to controls. Neurology Asia, 12 (1): 68, 2007

26- SURGES R., TAGGART P., SANDER J.W. and WALKER M.C.: Too long or too short? New insights into abnormal cardiac repolarization in people with chronic epilepsy and its potential role in sudden unexpected death. Epilepsia, 51 (5): 738-44, 2010.

27- SURGES R., SCOTT C.A. and WALKER M.C.: Enhanced QT shortening and persistent tachycardia after generalized seizures. Neurology, 74 (5): 421-6, 2010.

28- KRISHNAN V. and KRISHNAMURTHY K.B.: Interictal 12-lead electrocardiography in patients with epilepsy. Epilepsy Behav., 29 (1): 240-6, 2013.

29- AKALIN F., TIRTIR A. and YILMAZ Y.: Increased QT dispersion in epileptic children. Acta Paediatr., 92 (8): 916-20, 2003.

30- AL-NIMER M.S., AL-MAHDAWI S.A., ABDULLAH N.M. and AL-MAHDAWI A.: Epileptic Patients are at Risk of Cardiac Arrhythmias: A Novel Approach using QT-nomogram, Tachogram, and Cardiac Restitution Plots. J. Neurosci. Rural. Pract., 8 (1): 7-13, 2017.

31- LAMBERTS R.J., BLOM M.T., NOVY J., BELLUZZO M., SELDENRIJK A., PENNINX B.W., et al.: Increased prevalence of ECG markers for sudden cardiac arrest in refractory epilepsy. J. Neurol. Neurosurg. Psychiatry, 86 (3): 309-13, 2015.

32- SURGES R., JORDAN A. and ELGER C.E.: Ictal modulation of cardiac repolarization, but not of heart rate, is lateralized in mesial temporal lobe epilepsy. PLoS One, 8 (5): e64765, 2013.

33- STRAMBA-BADIALE M.: Epilepsy and the long QT syndrome: Is there a link?--Commentary on Hindocha et al. Epilepsia, 49 (2): 366, 2008.

34- AUERBACH D.S., McNITT S., GROSS R.A., ZAREBA W., DIRKSEN R.T. and MOSS A.J.: Genetic biomarkers for the risk of seizures in long QT syndrome. Neurology, 87 (16): 1660-8, 2016.

35- BROTHERSTONE R.1., BLACKHALL B. and McLELLAN A.: Lengthening of corrected QT during epileptic seizures. Epilepsia, 51 (2): 221-32, 2010.
36- SEYAL M., PASCUAL F., LEE C.Y., LI C.S. and BATEMAN L.M.: Seizure-related cardiac repolarization abnormalities are associated with ictal hypoxemia. Epilepsia, 52 (11): 2105-11, 2011.

37- BESTAWROS M., DARBAR D., ARAIN A., ABOUKHALIL B., PLUMMER W.D., DUPONT W.D., et al.: Ictal Asystole and Ictal Syncope: Insights into Clinical Management. Circ. Arrhythm. Electrophysiol., 8 (1): 15964, 2015.

38- NEI M.: Cardiac Effects of Seizures. Epilepsy Curr., 9 (4): 91-5, 2009

39- SURGES R.: The many facets of cardiac complications in epilepsy. J. Neurosci. Rural. Pract., 5 (1): 6-7, 2014.

40- SURGES R., MOSKAU S., VIEBAHN B., SCHOENEBAKE J.C., SCHWAB J.O. and ELGER C.E.: Prolonged atrial fibrillation following generalized tonic-clonic seizures. Seizure, 21 (8): 643-5, 2012.

41- STANDRIDGE S.M., HOLLAND K.D. and HORN P.S. Cardiac Arrhythmias and Ictal Events Within an Epilepsy Monitoring Unit. Pediatr. Neurol., 42 (3): 201-5, 2010.

42- ORAK M., USTÜNDAG M., GÜLO GLU C., OZHASENEKLER A., ALYAN O. and KALE E.: The role of the heart-type fatty acid binding protein in the early diagnosis of acute coronary syndrome and its comparison with troponin I and creatine kinase-MB isoform Am. J. Emerg. Med., 28 (8): 891-6, 2010.

43- HENNESSY M.J., LANGAN Y., ELWES R.D., BINNIE C.D., POLKEY C.E. and NASHEF L.: A study of mortality after temporal lobe epilepsy surgery. Neurology, Oct. 1, 53 (6): 1276, 1999.

44- NEI M., HO R.T., ABOU-KHALIL B.W., DRISLANE F.W., LIPORACE J., ROMEO A. and SPERLING M.R : EEG and ECG in sudden unexplained death in epilepsy. Epilepsia, Apr. 1, 45 (4): 338-45, 2004.

45- LANGAN Y., NASHEF L. and SANDER J.W.A.S.: Sudden unexpected death in epilepsy: a series of witnessed deaths. Journal of Neurology, Neurosurgery \& Psychiatry, 68 (2): 211-3, 2000.

46- CAMFIELD C.S., CAMFIELD P.R., GORDON K., WIRRELL E. and DOOLEY J.M.: Incidence of epilepsy in childhood and adolescence: A population-based study in Nova Scotia from 1977 to 1985 . Epilepsia, 37 (1): 19-23, 1996.

47- TIGARAN S. and DAM M.: Atrial fibrillation: an overlooked complication of epileptic seizures? Epilepsia, 39: 118,1998

48- NEI M., HO R.T. and SPERLING M.R.: EKG abnormalities during partial seizures in refractory epilepsy. Epilepsia, 41 (5): 542-8, 2000

49- BLUMHARDT L.D., SMITH P.E.M. and OWEN L.: Electrocardiographic accompaniments of temporal lobe epileptic seizures. The Lancet, 327 (8489): 1051-6, 1986.

50- SMITH P.E.M., HOWELL S.J.L., OWEN L. and BLUMHARDT L.D.: Profiles of instant heart rate during partial seizures. Electroencephalography and clinical neurophysiology, 72 (3): 207-17, 1989.

51- NATELSON B.H., SUAREZ R.V., TERRENCE C.F. and TURIZO R.: Patients with epilepsy who die suddenly 
have cardiac disease. Archives of neurology, 55 (6): 85760, 1998.

52- NATELSON B.H., SUAREZ R.V., TERRENCE C.F. and TURIZO R.: Patients with epilepsy who die suddenly have cardiac disease. Archives of neurology, 55 (6): 85760, 1998.

53- WOODRUFF B.K., BRITTON J.W., TIGARAN S., CASCINO G.D., BURRITT M.F., McCONNELL J.P. and JAFFE A.S.: Cardiac troponin levels following monitored epileptic seizures. Neurology, 60 (10): 1690-2, 2003.
54- TIGARAN S., MOLGAARD H., McCLELLAND R. DAM M. and JAFFE A.S.: Evidence of cardiac ischemia during seizures in drug refractory epilepsy patients. Neurology, 60: 492-5, 2003.

55- ALEHAN F., EROL I., CEMIL T., BAYRAKTAR N., OGÜS E. and TOKEL K.: Elevated CK-MB mass and plasma brain-type natriuretic peptide concentrations following convulsive seizures in children and adolescents: Possible evidence of subtle cardiac dysfunction. Epilepsia, 50 (4): 760-75, 2009.

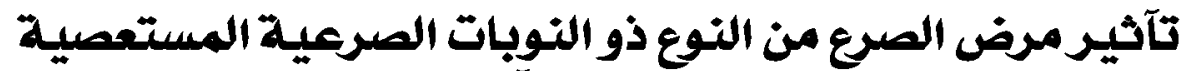

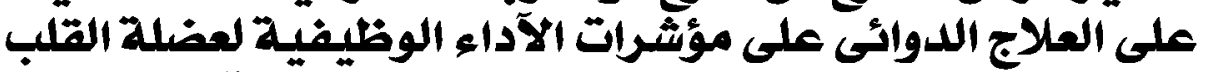

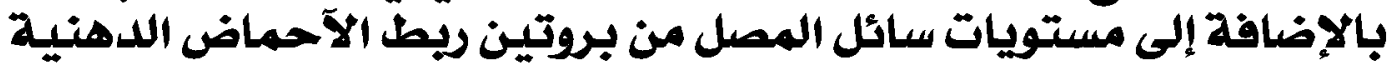 من النوع الخاص بعضلة من بروتين القلب}

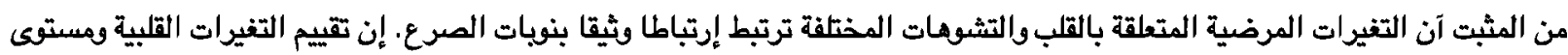

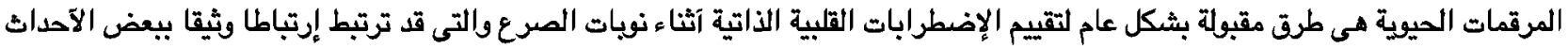
القلبية الوعائية وخاصة الموت القية القبى المفاجئ غير المتوقع.

الهدف: تقييم التغيرات القلبية ومستويات البلازما لبروتين ربط البروتين الدهنى من النوع الخاص بعضلة القلب (H-FABP) فى مرضى الآطفال المصابين بصرع مقاوم اللعلاج.

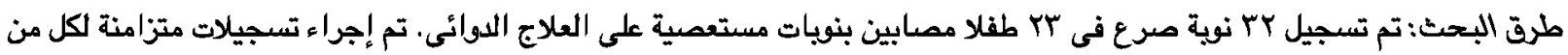

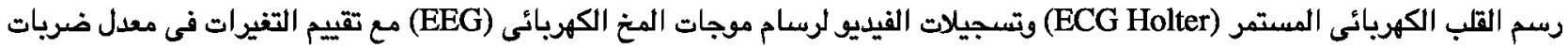

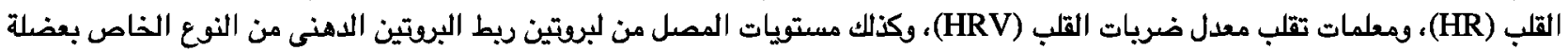

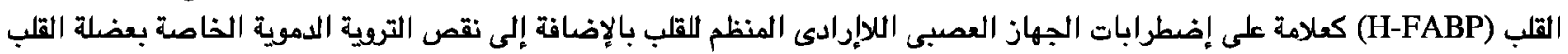

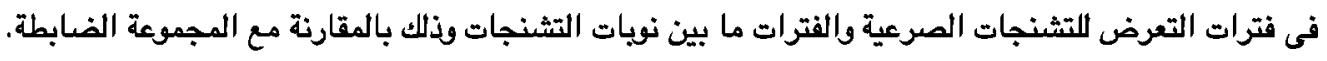

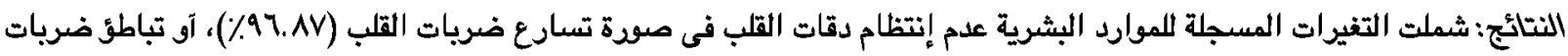

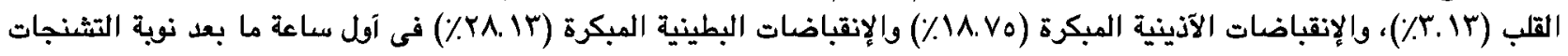

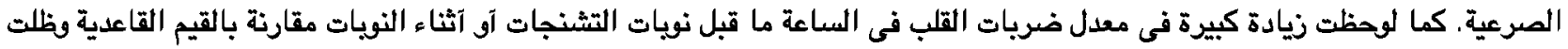

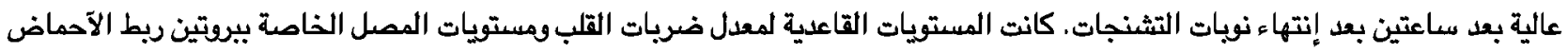

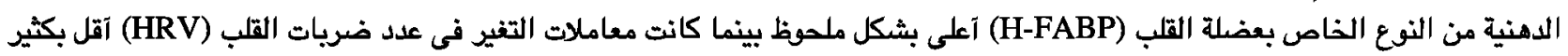
فى المرضى مقارنة بالمجموعة الضابطة.

الخلاصة: قد تحدث تغيرات قلبية مامة حتى قبل بداية النوبة فى حالات الصرع المستعصية. قد يشير إرتفاع مستويات بروتين ربط

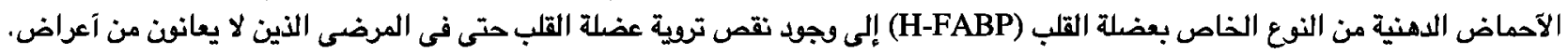

